\title{
The Nonlinear Maslov index and the Calabi homomorphism
}

\author{
Gabi Ben Simon * \\ School of Mathematical Sciences \\ Tel Aviv University 69978 Tel Aviv, Israel \\ gabiben@post.tau.ac.il
}

September 28, 2018

\begin{abstract}
In this paper we find that the asymptotic nonlinear Maslov index defined on the universal cover of the group of all contact Hamiltonian diffeomorphisms of the standard $2 n-1$ dimensional contact sphere is a quasimorphism. Then we show our main result: Let $M$ be standard $n-1$ complex projective space. We prove that the value of the pull back of the asymptotic nonlinear Maslov index to the universal cover of the group of Hamiltonian diffeomorphisms of $M$, restricted to elements supported in sufficiently small open subsets of $M$, equals $\frac{-n}{2 \pi v o l(M)}$ times the Calabi invariant of these elements.
\end{abstract}

\section{Introduction and results}

\subsection{Basics on the nonlinear Maslov index}

Let $\left(S^{2 n-1}, \xi\right)$ be the $2 n-1$ dimensional sphere endowed with the standard contact structure. We denote by $\mathcal{C}$ the identity component of the group of those contact Hamiltonian diffeomorphisms of the contact manifold $\left(S^{2 n-1}, \xi\right)$ which commute with the involution map, $x \mapsto-x$, of the sphere (see 7] for preliminaries on contact manifolds). Let $\widetilde{\mathcal{C}}$ be the universal cover of $\mathcal{C}$. Recall that an element $\tilde{f}$ of $\widetilde{\mathcal{C}}$ can be identified as the time-1-map of a flow generated by a contact Hamiltonian, up to a homotopy relation between the paths generating $\tilde{f}$. Moreover, $\mathcal{C}$ carries the structure of an infinite dimensional manifold. Following Givental (see [5]), we call discriminant

*This paper is a part of the author's Ph.D. thesis, being carried out under the supervision of Professor Leonid Polterovich, at Tel-Aviv University. 
the hypersurface of $\mathcal{C}$ formed by all contactomorphisms which have a fixed point, and preserve the standard contact form at that point, we denote it by $\Delta(\mathcal{C})$. Using generating functions methods Givental has defined a co-orientation of $\Delta(\mathcal{C})$ in $\mathcal{C}$.

Given a path $\left\{\tilde{f}_{t}\right\}_{t \geq 0}$ in $\mathcal{C}$, an intersection index can be assigned to this path by counting all signed intersections, relative to the co-orientation and at non singular points of $\Delta(\mathcal{C})$, with a subspace of $\Delta(\mathcal{C})$ called the train, denoted by $\Gamma(\mathcal{C})$, see again [5].

We call the intersection index of $\left\{\tilde{f}_{s}\right\}_{s \in[0, t]}$ with $\Gamma(\mathcal{C})$ the non linear Maslov index of $\left\{\tilde{f}_{s}\right\}_{s \in[0, t]}$ and denote it by $\mu(t)$. Following Givental we now define the asymptotic nonlinear Maslov index.

Let $\tilde{f}$ be an element of $\widetilde{\mathcal{C}}$ generated by a time- 1 periodic contact Hamiltonian $\widetilde{F}: S^{2 n-1} \times S^{1} \longrightarrow \mathbb{R}$. Let $\left\{\tilde{f}_{t}\right\}_{t \geq 0}$ be the flow generated by $\widetilde{F}$ which is a path in $\mathcal{C}$. We define the asymptotic nonlinear Maslov index of $\tilde{f}$ as

$$
m(\tilde{f}):=\lim _{t \rightarrow \infty} \frac{\mu(t)}{t}
$$

\subsection{The quasimorphism property}

Definition 1.1. Let $G$ be a group. A quasimorphism $r$ on $G$ is a function $r: G \rightarrow \mathbb{R}$ which satisfies the homomorphism equation up to a bounded error: there exists $R>0$ such that

$$
|r(f g)-r(f)-r(g)| \leq R
$$

for all $f, g \in G$.

See 2 for preliminaries on quasimorphisms. A quasimorphism $r_{h}$ is called homogeneous if $r_{h}\left(g^{m}\right)=m r_{h}(g)$ for all $g \in G$ and $m \in \mathbb{Z}$. Every quasimorphism $r$ gives rise to a homogeneous one

$$
r_{h}(g)=\lim _{m \rightarrow \infty} \frac{r\left(g^{m}\right)}{m} .
$$

We now can state the first theorem of this paper which is actually an easy consequence of $[5]$.

Theorem 1.2. The asymptotic nonlinear Maslov index $m: \widetilde{\mathcal{C}} \longrightarrow \mathbb{R}$ is a quasimorphism.

The proof will be given in Section 2 after some preliminaries on the asymptotic nonlinear Maslov index.

\subsection{The pull back of $\mathrm{m}$}

Let $\left(\mathbb{C} P^{n-1}, \omega\right)$ be $n-1$ dimensional complex projective space endowed with the Fubini-Study form 
normalized by $\int_{\mathbb{C} P^{1}} \omega=\pi$.

Let $G=\operatorname{Ham}\left(\mathbb{C} P^{n-1}, \omega\right)$ be the group of the identity component of all Hamiltonian diffeomorphisms of $\left(\mathbb{C} P^{n-1}, \omega\right)$ and $\widetilde{G}$ its universal cover (see [7] and [8] for preliminaries on $G$ ). Let $\left\{f_{t}\right\}_{t \in[0,1]}$ be a path in $G$ representing an element of $\widetilde{G}$. For such an element there is a unique generating Hamiltonian $F: \mathbb{C} P^{n-1} \times S^{1} \longrightarrow \mathbb{R}$ satisfing the normalization condition:

$$
\int_{\mathbb{C} P^{n-1}} F_{t} \omega^{n-1}=0
$$

for all $t \in S^{1}$.

We denote the space of all normalized Hamiltonians by F. In order to pull back the asymptotic nonlinear Maslov index from $\widetilde{\mathcal{C}}$ to $\widetilde{G}$ we will use the Hopf bundle

$$
p: S^{2 n-1} \longrightarrow \mathbb{C} P^{n-1} .
$$

Let $\left\{f_{t}\right\}_{t \in[0,1]}$ be a representative of an element of $\widetilde{G}$, and let $F \in \mathrm{F}$ be the unique normalized Hamiltonian generating this path. Then we can lift $F$ to $S^{2 n-1}$ to a time dependent function,

$$
\widetilde{F}_{t}:=p^{*} F_{t} .
$$

In other words $\widetilde{F}$ is constant along the fibers of the fiber bundle $p: S^{2 n-1} \longrightarrow \mathbb{C} P^{n-1}$. This way $\widetilde{F}$ is a contact Hamiltonian on the contact sphere lifting the Hamiltonian $F$. The contact flow $\left\{\tilde{f}_{t}\right\}_{t \in[0,1]}$ generated by this contact Hamiltonian constitutes a lift of the flow $\left\{f_{t}\right\}_{t \in[0,1]}$, and represents an element of $\widetilde{\mathcal{C}}$. As such it has an asymptotic nonlinear Maslov index, which induces an asymptotic nonlinear Maslov index on $\widetilde{G}$. We will denote by

$$
i: \widetilde{G} \longrightarrow \widetilde{\mathcal{C}}
$$

the above injection, and by

$$
i^{*} m: \widetilde{G} \longrightarrow \mathbb{R}
$$

the pullback of the asymptotic nonlinear Maslov index. As a corollary of Theorem 1.2 we have

Corollary 1.3. The pull back of the asymptotic nonlinear Maslov index:

$$
i^{*} m: \widetilde{G} \longrightarrow \mathbb{R}
$$

is a quasimorphism.

Remark. Any Hamiltonian (not necessarily normalized) generating an element of $\widetilde{G}$ can be lifted via $p$ in the way described above. The normalization condition is required since we want to embed $\widetilde{G}$ in $\widetilde{\mathcal{C}}$.

We close this subsection with a simple but an important example. 
Example 1.4. Consider the flow $f_{t}=\mathbf{1}$ for all $t$ where 1 is the identity map of $\mathbb{C} P^{n-1}$. Any function of the form $F(x, t)=F(t)$ (clearly, not necessarily normalized) will generate this flow. Let $p^{*} F_{t}$ be its lift to the sphere. An easy computation will show that the flow $\left\{\tilde{f}_{t}\right\}$, generated by $p^{*} F_{t}$, leaves the fibers of the Hopf bundle invariant, and rotates them in an angle $e^{i F(t)}$ for time $t$.

\subsection{The Calabi property of $i^{*} \mathbf{m}$}

In this subsection we will assume that $G=\operatorname{Ham}(M, \omega)$, where $M^{2 n}$ is any closed connected symplectic manifold.

The group $G$ has a natural class of subgroups $G_{U}$ associated to non empty open subsets $U \subset M$. An element of $G$ lies in $G_{U}$ if and only if it can be generated by a Hamiltonian flow $\left\{f_{t}\right\}_{t \in[0,1]}$ with $f_{0}=\mathbf{1}$, generated by a Hamiltonian $F_{t}$ satisfying the following condition:

$$
F_{t}: M \longrightarrow \mathbb{R}, t \in[0,1]
$$

such that

$$
\operatorname{support}\left(F_{t}\right) \subset U \text { for all } t \text {. }
$$

Consider the map $\mathrm{Cal}_{U}: G_{U} \longrightarrow \mathbb{R}$ given by:

$$
f \mapsto \int_{0}^{1} d t \int_{M} F_{t} \omega^{n}
$$

When the symplectic form $\omega$ is exact on $U$, this map is well defined, meaning that it does not depend on the specific choice of the Hamiltonian $F$ generating $f$. In fact, $\mathrm{Cal}_{U}$ is a homomorphism called the Calabi homomorphism (see [1, 3] and [10 for further information).

The above definitions can be "lifted" to $\widetilde{G}$ : for a non empty subset $U \subset M$, consider the subgroup $\widetilde{G_{U}} \subset \widetilde{G}$ defined as follows. An element of $\widetilde{G}$ lies in $\widetilde{G_{U}}$ if and only if it can be represented by a Hamiltonian flow $\left\{f_{t}\right\}_{t \in[0,1]}$, with $f_{0}=\mathbf{1}$ generated by a Hamiltonian $F_{t}$ satisfying condition (44). Formula (5) gives rise to a well defined homomorphism

$$
\widetilde{C a l}: \widetilde{G_{U}} \longrightarrow \mathbb{R}
$$

In what follows we deal with the class $\mathcal{D}$ of all non empty open subsets $U$ which can be displaced by a Hamiltonian diffeomorphism:

$$
h U \cap \operatorname{Closure}(U)=\varnothing \text { for some } h \in G \text {. }
$$

We need one more definition before stating our main result.

Definition 1.5. A quasimorphism on $\widetilde{G}$ coinciding with the Calabi homomorphism $\left\{\widetilde{C a l}_{U}: \widetilde{G}_{U} \rightarrow \mathbb{R}\right\}$ on any $U \in \mathcal{D}$ is said to have the Calabi property.

From now on $M=\mathbb{C} P^{n-1}$. Here is our main result: 
Theorem 1.6. The pull back of the asymptotic nonlinear Maslov index $i^{*} m: \widetilde{G} \longrightarrow \mathbb{R}$ has the Calabi property up to a multiplication by $\frac{-n}{2 \pi v o l(M)}$. That is

$$
i^{*} m([f])=\frac{-n \widetilde{\operatorname{Cal}}([f])}{2 \pi \operatorname{vol}(M)}
$$

for every element $[f] \in \widetilde{G}_{U}$.

The proof will be given in section 3 .

Remark. Given a Hamiltonian $H: M \times S^{1} \longrightarrow \mathbb{R}$ denote by $\left\{f_{t}^{H}\right\}_{t \in[0,1]}$ the element in $\widetilde{G}$ generated by $H$. Suppose that a function $r: \widetilde{G} \longrightarrow \mathbb{R}$ satisfies the following condition: if a sequence $\left\{H_{i}\right\}$ of smooth (possibly time dependent) Hamiltonians $H_{i}: M \times S^{1} \longrightarrow \mathbb{R}$ converges $C^{0}$ uniformly to a smooth function $H: M \times S^{1} \longrightarrow \mathbb{R}$ then

$$
r\left(f_{t}^{H_{i}}\right) \longrightarrow r\left(f_{t}^{H}\right) \text { as } i \rightarrow \infty
$$

In such case the function $r$ will be called continuous.

It follows from Corollary 3 of Section 9 of [5] that the pull back of the asymptotic nonlinear Maslov index is continuous. Thus, we get: the pull back of the asymptotic nonlinear Maslov index $i^{*} m: \widetilde{G} \longrightarrow \mathbb{R}$ is a continuous quasimorphism with the Calabi property (up to a multiplication by a constant).

\subsection{Other quasimorphisms with the Calabi property}

Another construction of a continuous quasimorphism on $\widetilde{G}$ with the Calabi property has been suggested by Entov and Polterovich in [4. In particular this construction applies to the manifold $\left(\mathbb{C} P^{n-1}, \omega\right)$.

Their construction uses the spectral invariants of elements of $\widetilde{G}$. These spectral invariants are defined in terms of the quantum and Floer theory of the symplectic manifold. The first step in our proof of theorem 1.6 follows the lines of [4. Moreover, step 3 of the proof of 1.6 was motivated, partly, by the inspection of the behavior of spectral invariants defined in terms of the theory of generating functions (see [4] for more details).

Acknowledgments. My sincere gratitude goes to Leonid Polterovich for all his support, ideas, and encouragement. I want to thank Michael Entov for reading drafts of this paper, and for coming to my talk about this work at Tel Aviv university and shearing with me his opinion. I thank also Yaron Ostrover for reading the last draft of this paper and for his remarks. 


\section{Preliminaries on the nonlinear Maslov index}

In this short section we state the main properties of the asymptotic nonlinear Maslov index and the nonlinear Maslov index $\mu$, which we use in the proof of the main result (theorem 1.6).

First, we recall the reader that the sign of intersection of a path with the train ( which is a subspace of the discriminant) is determined due to a co-orientation of the train defined by generating functions methods. The two basic facts that we need are grouped together in the following theorem which is due to Givental (see [5], [6], and [9]).

Theorem 2.1. (a) The nonlinear Maslov index relative to the train of any two paths in $\mathcal{C}$ is the same provided that there is a homotopy between these two paths such that the end points of the paths of this homotopy are maps without fixed points on the sphere.

(b) Let $f$ be a Hamiltonian map of $\mathbb{C} P^{n-1}$ and let $\tilde{f}$ be its lift to $\mathcal{C}$. Consider $\tilde{f}$ as a point in $\mathcal{C}$ and assume that it has no fixed points. Then the nonlinear Maslov index of the loop $\left\{e^{ \pm 2 \pi i t} \tilde{f}\right\}_{t \in[0,1]}$ with the train is $\pm n$.

\subsection{Proof of Theorem 1.2}

We are now in position to prove Theorem [1.2 :

Proof of Theorem 1.2. Let $\tilde{f} \in \widetilde{C}$. By definition, it is generated by a time-1 periodic contact Hamiltonian. Let $\left\{\tilde{f}_{t}\right\}$ be the contact flow generated by this Hamiltonian. Then we have $\tilde{f}^{m}=\tilde{f}_{m}$ for all $m$ integer. This means that

$$
m(\tilde{f})=\lim _{m \rightarrow \infty} \frac{\mu\left(\tilde{f}^{m}\right)}{m}
$$

It is enough to prove that $\mu$ is a quasimorphism on $\widetilde{\mathcal{C}}$ (see section 1.2). For this, let $[\tilde{f}]=\left\{\tilde{f}_{t}\right\}_{t \in[0,1]},[\tilde{h}]=\left\{\tilde{h}_{t}\right\}_{t \in[0,1]}$ be elements of $\widetilde{\mathcal{C}}$. Consider the following path which represents $[\tilde{f}][\tilde{h}]$. It is the concatenation:

$$
\left\{\tilde{f}_{2 t}\right\}_{0 \leq t \leq \frac{1}{2}} *\left\{\tilde{f}_{1} \tilde{h}_{2 t-1}\right\}_{\frac{1}{2} \leq t \leq 1}:= \begin{cases}\tilde{f}_{2 t} & 0 \leq t \leq \frac{1}{2} \\ \tilde{f}_{1} \tilde{h}_{2 t-1} & \frac{1}{2} \leq t \leq 1\end{cases}
$$

We denote this path by $\gamma$.

It follows from Givental's construction that $\mu$ is additive with respect to concatenation. So we have that

$$
\mu(\gamma)=\mu\left(\left\{\tilde{f}_{2 t}\right\}\right)+\mu\left(\left\{\tilde{f}_{1} \tilde{h}_{2 t-1}\right\}\right) .
$$


Moreover it is also follows from the construction that

$$
\left|\mu\left(\left\{\tilde{h}_{2 t-1}\right\}\right)-\mu\left(\left\{\tilde{f}_{1} \tilde{h}_{2 t-1}\right\}\right)\right| \leq 2 n .
$$

Combining (9) and (10) we get

$$
\left|\mu(\gamma)-\mu\left(\left\{\tilde{f}_{2 t}\right\}\right)-\mu\left(\left\{\tilde{h}_{2 t-1}\right\}\right)\right| \leq 2 n .
$$

Since the paths $\left\{\tilde{f}_{2 t}\right\}$ and $\left\{\tilde{h}_{2 t-1}\right\}$ represent respectively $[\tilde{f}]$ and $[\tilde{h}]$ we have:

$$
|\mu([\tilde{f}][\tilde{h}])-\mu([\tilde{f}])-\mu([\tilde{h}])| \leq 2 n
$$

which is what we need.

\section{Proof of Theorem 1.6}

\section{$3.1 \quad$ Step 1}

Let $[f]=\left\{f_{t}\right\}_{t \in[0,1]}$ be a representative of an element in $\widetilde{G}_{U}$ for some open set $U$ in $\mathcal{D}$, generated by a time-1 periodic Hamiltonian $\left\{F_{t}\right\}$ (see 1.4). We have to show that:

$$
\frac{-n \widetilde{\operatorname{Cal}}([f])}{2 \pi \operatorname{vol}(M)}=i^{*} m([f]) \text {. }
$$

Recall that actually the r.h.s is the asymptotic nonlinear Maslov index of the lift $\left\{\tilde{f}_{t}\right\}_{t \geq 0}$ of the flow $\left\{f_{t}\right\}_{t \geq 0}$ (see 1.1 and 1.3). As a first step we claim that it is enough to show that the asymptotic nonlinear Maslov index of the path $\left\{\widetilde{h f}_{t}\right\}_{t \geq 0}$ which is a lift of the path $\left\{h f_{t}\right\}_{t \geq 0}$ (where $h$ is the displacement element of $U$ ) equals the 1.h.S of (11). this follows easily from inequality (10). Since we get the difference of the nonlinear Maslov indexes of these paths is bounded by $2 n$, thus they have the same asymptotic nonlinear Maslov index.

¿From now on we will focus on the concatenation:

$$
\left\{h_{t}\right\}_{t \in[0,1]} *\left\{h f_{t-1}\right\}_{t \geq 1}:=\gamma_{t}
$$

and its lift to $\mathcal{C}$ :

$$
\left\{\tilde{h}_{t}\right\}_{t \in[0,1]} *\left\{\widetilde{h f}_{t-1}\right\}_{t \geq 1}:=\widetilde{\gamma}_{t}
$$

It is important to emphasize, that the paths $\left\{\gamma_{t}\right\}_{0 \leq t \leq t_{0}}$ and $\left\{\widetilde{\gamma}_{t}\right\}_{0 \leq t \leq t_{0}}\left(\right.$ for $t_{0} \geq 1$ ) represent respectively $\left\{h f_{t}\right\}_{0 \leq t \leq t_{0}}$ and $\left\{\widetilde{h f}_{t}\right\}_{0 \leq t \leq t_{0}}$ as elements of $\widetilde{G}$ and $\widetilde{\mathcal{C}}$ respectively.

Second, note that the set of fixed points of $\left\{h f_{t}\right\}_{t \geq 0}$ coincides with the set of fixed points of $h$ for all $t \geq 0$. This is because $h$ displaces $U$. Let $x_{0}$ be a fixed point of $h$, and let $\widetilde{x_{0}} \in p^{-1}\left(x_{0}\right)$ be a point on the fiber $p^{-1}\left(x_{0}\right)$ above $x_{0}$. Of course $\widetilde{\gamma}_{t}\left(\widetilde{x_{0}}\right)$ is a 
lift of $\gamma_{t}\left(x_{0}\right)$. In particular for $0 \leq t \leq 1$ the path $\widetilde{\gamma}_{t}\left(\widetilde{x_{0}}\right)$ is a lift of the loop $\gamma_{t}\left(x_{0}\right)$ which is not necessarily closed. Nevertheless the point $\widetilde{x_{0}}$ will return to its fiber for $t=1$. For $t \geq 1$ we have $\gamma_{t}\left(x_{0}\right)=x_{0}$ so the fiber $p^{-1}\left(x_{0}\right)$ is invariant under the flow $\widetilde{\gamma}_{t}$. Therefore we have:

$$
\widetilde{\gamma}_{t}\left(\widetilde{x_{0}}\right)=e^{i \theta(t)} \widetilde{x_{0}}, t \geq 1
$$

where $\theta(t)$ is determined by the contact flow generated by the lift of the Hamiltonian generating the path $\gamma_{t}$.

\section{$3.2 \quad$ Step 2}

Now, assume that for $t=s, s>1$ the path $\widetilde{\gamma}_{t}$ intersects the train $\Gamma(\mathcal{C})$. This means that there exists $x_{0} \in\{f i x(h)\}$ such that in equation (12) we have $\theta(s)=2 \pi k$ for some $k \in \mathbb{Z}$. In the second step of the proof we first calculate the function $\theta(s)$, and then make a reduction of the path $\widetilde{\gamma}_{t}$ to a path in which the function $\theta(s)$ is linear. This will help us to make the finale reductive step in the next section in which we actually calculate the asymptotic nonlinear Maslov index.

Notation: From now on we denote $\frac{-\widetilde{\operatorname{Cal}([f])}}{\operatorname{vol}(M)}$ by $C$.

Let $\left\{K_{t}\right\}$ be the normalized Hamiltonian generating the flow $\left\{\gamma_{t}\right\}$, and let $\left\{\widetilde{K}_{t}\right\}$ be its lift. Clearly for $t \geq 1$ we have:

$$
K_{t}=F_{t}+c(t)
$$

where $F_{t}$ is a time- 1 periodic Hamiltonian generating the flow $\left\{f_{t}\right\}, M$ is $\mathbb{C} P^{n-1}$ and $c(t)=\frac{-1}{\operatorname{vol} M} \int_{M} F_{t} \omega^{n-1}$. Moreover for all $x \in M \backslash U$ we have $K_{t}(x)=c(t)$.

Define for $s \geq 1$

$$
\theta(s):=\int_{1}^{s} c(t) d t=-\int_{1}^{s} d t \frac{1}{\operatorname{vol} M} \int_{M} F_{t} \omega^{n-1} .
$$

Since $F_{t}$ is periodic we have for every $m \in \mathbb{N}$ and $0 \leq v \leq 1$

$$
\theta(m+v)=C(m-1)-\int_{0}^{v} d t \frac{1}{\operatorname{vol} M} \int_{M} F_{t} \omega^{n-1}
$$

Note also that the function $\varphi(s):=\theta(s)-C(s-1)$ is time-1 periodic and satisfies $\varphi(m)=0$ for every $1 \leq m \in \mathbb{N}$. In particular for all $m \in \mathbb{N}$ we have

$$
C(m-1)=\theta(m)
$$

Now recall that for every $\tilde{x}_{j}$ above $x_{j} \in\{f i x(h)\}$ the function $\theta(s)$ equals the time dependent constant defined by equation (12). 
In other words $\theta(s)$ measures the total displacement of the fiber at time $s$. We can assume with out the loss of generality, from now on, that $h$ has a finite number of fixed points. This can be achieved by a small perturbation of $h$. We will say that $x_{0} \in\{f i x(h)\}$ occurs at $t=s_{0}$ if $\widetilde{\gamma}_{s_{0}}$ is the identity map if restricted to the fiber $p^{-1}\left(x_{0}\right)$. Now let $\left\{x_{1} \ldots x_{l}\right\}$ be the set of fixed points of $h$ and let $\tilde{x}_{j}$ be a point above $x_{j}$ for all $1 \leq j \leq l$. Let $\alpha_{j}, 0 \leq \alpha_{j}<2 \pi$, be the angle defined by the equation $\widetilde{\gamma}_{1}\left(\tilde{x}_{j}\right)=e^{i \alpha_{j}} \tilde{x}_{j}$. We have for $s \geq 1$

$$
\widetilde{\gamma}_{s}\left(\tilde{x}_{j}\right)=e^{i C(s-1)} e^{i \varphi(s)} e^{i \alpha_{j}}\left(\tilde{x}_{j}\right)
$$

From the properties of $\varphi(s)$ we get that the path $\left\{e^{i \varphi\left(s_{0}+t\right)}\right\}_{t \in[0, k]}, k \in \mathbb{N}$ is homotopic to the constant loop $\left\{e^{i \varphi\left(s_{0}\right)}\right\}$ for every $s_{0} \geq 0$. We emphasize that the homotopy has $\left\{e^{i \varphi\left(s_{0}\right)}\right\}$ as the starting and ending point of all of its paths.

Let us denote this homotopy by $\left\{e^{i F_{s}(t)}\right\}_{(s, t) \in[0,1] \times[0, k]}$. So we have

$$
F_{0}(t)=\varphi\left(s_{0}+t\right), F_{1}(t)=\varphi\left(s_{0}\right)
$$

and

$$
F_{s}(0)=F_{s}(k)=\varphi\left(s_{0}\right) .
$$

For reasons that will turn out to be clear we are looking for $s_{0}$ that will satisfy the following condition

$$
\begin{gathered}
C\left(s_{0}+k-1\right)+\varphi\left(s_{0}+k\right)+\alpha_{j} \notin 2 \pi \mathbb{Z} \\
\text { for all } 1 \leq j \leq l \text { and } k \in \mathbb{N} .
\end{gathered}
$$

Such $s_{0}$ clearly exists. For this, first note that the set $2 \pi \mathbb{Z}$ is countable as well as $\mathbb{N}$, the set of $\left\{\alpha_{1} \ldots \alpha_{l}\right\}$ is finite, and finally $\varphi\left(s_{0}+k\right)=\varphi\left(s_{0}\right)$ for all $k \in \mathbb{N}$. So our problem is to find a point in the image of the function $C(s-1)+\varphi(s)$ which belongs to the complement of the countable set $\bigcup_{j, k}\left\{2 \pi \mathbb{Z}-\alpha_{j}-k C\right\}$. This function being unbounded, clearly contains a segment under its image, and thus a point in the complement of the set above.

For such $s_{0}$ we have for all $j, k$

$$
\widetilde{\gamma}_{s_{0}+k}\left(\tilde{x}_{j}\right)=e^{i C\left(s_{0}+k-1\right)} e^{i \varphi\left(s_{0}\right)} e^{i \alpha_{j}}\left(\tilde{x}_{j}\right) \neq \tilde{x}_{j}
$$

That is $\widetilde{\gamma}_{s_{0}+k}$ does not have fixed points for all $k \in \mathbb{N}$ (and thus it is outside of the train). Now we have come to the key idea of step 2.

Lemma 3.1. The family of maps

$$
\psi(s, t)=\left\{\widetilde{\gamma}_{s_{0}+t} e^{-i F_{s}(t)} e^{i \varphi\left(s_{0}\right)}\right\}_{(s, t) \in[0,1] \times[0, k]}
$$

is a homotopy of paths in $\mathcal{C}$ which has the following properties: 
(a) For $s=1$ it is the path $\left\{\widetilde{\gamma}_{s_{0}+t}\right\}_{t \in[0, k]}$

(b) The starting point and the end point of all of the paths of this homotopy does not have fixed points. That is, the paths which are obtained by substituting $t=0$ and $t=k$ do not have fixed points.

Proof. For $s=1$ we have $F_{1}(t)=\varphi\left(s_{0}\right)$. Thus by the very definition of $\psi$ we get part (a). For $t=0$ and $t=k$ we know that for every $0 \leq s \leq k F_{s}(0)=F_{s}(k)=\varphi\left(s_{0}\right)$. Thus by the very definition of $\psi$ and formula (16) we get part (b) of the lemma.

It follows from the invariance of the nonlinear Maslov index with respect to homotopy (see theorem 2.1) that the path $\psi(0, t)$ has the same index as the path $\psi(1, t)$ (which is $\left.\left\{\widetilde{\gamma}_{s_{0}+t}\right\}_{t \in[0, k]}\right)$. Now, restricting the path $\psi(0, t)$ to the fibers over the fixed points we have the following equation

$$
\psi(0, t)\left(\tilde{x}_{j}\right)=e^{i C\left(s_{0}+t-1\right)} e^{i \varphi\left(s_{0}\right)} e^{i \alpha_{j}}\left(\tilde{x}_{j}\right)
$$

We get the following equation by the restriction of the path $e^{i\left[C-\varphi\left(s_{0}\right)\right]} \psi(0, t)$ to the fibers over the fixed points:

$$
e^{i\left[C-\varphi\left(s_{0}\right)\right]} \psi(0, t)\left(\tilde{x}_{j}\right)=e^{i C\left(s_{0}+t\right)} e^{i \alpha_{j}}\left(\tilde{x}_{j}\right)
$$

Moreover, we get from equation (10) that the difference between the nonlinear Maslov indexes of $e^{i\left[C-\varphi\left(s_{0}\right)\right]} \psi(0, t)$ and $\psi(0, t)$ is bounded by $2 n$ for all $k \in \mathbb{N}$. Thus, by the same argument we have used in step 1, we get that the asymptotic nonlinear Maslov indexes of the paths $e^{i\left[C-\varphi\left(s_{0}\right)\right]} \psi(0, t)$ and $\psi(0, t), t \geq 0$, are equal.

It follows from the discussion above that without the loss of generality we can assume For $s \geq s_{0}, s_{0} \geq 1$ that:

$$
\widetilde{\gamma}_{s}\left(\tilde{x}_{j}\right)=e^{i\left[C\left(s-s_{0}\right)+\theta_{j}\right]}\left(\tilde{x}_{j}\right)
$$

where $\theta_{j}$ is the initial condition depends on $s_{0}$ and $x_{j} 0 \leq \theta_{j}<2 \pi$ defined by the equation

$$
\widetilde{\gamma}_{s_{0}}\left(\tilde{x}_{j}\right)=e^{i \theta_{j}}\left(\tilde{x}_{j}\right)
$$

\subsection{Step 3}

Before introducing the last step of the proof let us motivate it. We have seen that we may assume that the fibers above the fixed points of $h$ rotate at the same angular speed and in the same direction of rotation (where the orientation is induced by the action of the group $\left.\left\{e^{i t}\right\}\right)$. So within an interval of length $\frac{2 \pi}{|C|}$ all the above fibers makes full rotation. Since this is a periodic phenomena this might lead us to believe 
that the number of nonlinear Maslovs with the train is fixed for each interval of length $\frac{2 \pi}{|C|}$ (provided that the starting point of such a path is outside of the train). To show that this is the actual situation we need the following lemma.

Lemma 3.2. Let $\left\{\widetilde{\gamma}_{t}\right\}_{t \geq 1}$ be the path in $\mathcal{C}$ as defined at the beginning of this section where we assume it satisfies equation (18). Choose $s_{0}$ such that $\widetilde{\gamma}_{s_{0}}$ is without fixed points. Then the family of maps

$$
\left\{e^{C i(1-s) t} \widetilde{\gamma}_{s_{0}+s t}\right\}_{(s, t) \in[0,1] \times\left[0, \frac{2 \pi}{|C|}\right]}
$$

is a homotopy between the paths $\left\{\widetilde{\gamma}_{s_{0}+t}\right\}_{t \in\left[0, \frac{2 \pi}{|C|}\right]}$ and $\left\{e^{C i t} \widetilde{\gamma}_{s_{0}}\right\}_{t \in\left[0, \frac{2 \pi}{|C|}\right]}$ such that the end points of the paths of this homotopy stay outside of the discriminant.

Proof. The first part of the lemma is trivial since all we need to do is to substitute $s=0$ and $s=1$ to get the above paths. It is also obvious that the homotopy stays in $\mathcal{C}$. What we need to show is that the constant path

$$
\Lambda_{1}^{s}:=\left\{\widetilde{\gamma}_{s_{0}}\right\}
$$

and the path

$$
\Lambda_{2}^{s}:=\left\{e^{C i(1-s) \frac{2 \pi}{|C|}} \widetilde{\gamma}_{s_{0}+\frac{2 \pi s}{|C|}}\right\}_{s \in[0,1]}
$$

does not have any fixed points.

We need only to check it for the path $\Lambda_{2}$. So let $x_{0}$ be any point of $\{f i x(h)\}$, and let $\tilde{x}_{0}$ be any point above $x_{0}$. Let $0<\theta_{0}<2 \pi$ be the angle of rotation of $\tilde{x}_{0}$ at time $s_{0}$, chosen as in equation (18). According to equation (18) we have

$$
\widetilde{\gamma}_{s_{0}+\frac{2 \pi s}{|C|}}\left(\tilde{x}_{0}\right)=e^{i\left(\operatorname{sign}(C) 2 \pi s+\theta_{0}\right)}\left(\tilde{x}_{0}\right)
$$

from this we get

$$
\Lambda_{2}^{s}\left(\tilde{x}_{0}\right)=e^{i\left[\operatorname{sign}(C)(1-s) 2 \pi+\operatorname{sign}(C) 2 \pi s+\theta_{0}\right]}\left(\tilde{x}_{0}\right)
$$

or simply:

$$
\Lambda_{2}^{s}\left(\tilde{x}_{0}\right)=e^{i\left(\operatorname{sign}(C) 2 \pi+\theta_{0}\right)}\left(\tilde{x}_{0}\right) .
$$

According to our choice of $\theta_{0}$ clearly $\Lambda_{2}^{s}\left(\tilde{x}_{0}\right) \neq \tilde{x}_{0}$ for all $s \in[0,1]$. This concludes the proof of our lemma.

Here we use again part $(a)$ of theorem 2.1. More accurately, we get from the theorem according to lemma 3.2 , that the paths

$$
\left\{\widetilde{\gamma}_{s_{0}+t}\right\}_{t \in\left[0, \frac{2 \pi}{|C|}\right]}
$$

and

$$
\left\{e^{C i t} \widetilde{\gamma}_{s_{0}}\right\}_{t \in\left[0, \frac{2 \pi}{|C|}\right]}
$$

has the same nonlinear Maslov index with the train. The calculation of the nonlinear Maslov index of $\left\{e^{C i t} \widetilde{\gamma}_{s_{0}}\right\}_{t \in\left[0, \frac{2 \pi}{|C|}\right]}$ follows from part $(b)$ of theorem 2.1 from which we get that 
Corollary 3.3. The nonlinear Maslov index of the path $\left\{\widetilde{\gamma}_{s_{0}+t}\right\}_{t \in\left[0, \frac{2 \pi}{|C|}\right]}$ with the train is $\operatorname{sign}(C) n$.

Now we can calculate the asymptotic nonlinear Maslov index of $[f]$. Choose $s_{0} \geq 1$ such that $\widetilde{\gamma}_{s_{0}}$ does not have any fixed points. Note that $\widetilde{\gamma}_{s_{0}+\frac{2 \pi k}{|C|}}$ does not have fixed points, and thus it is outside of the train, for all $k \in \mathbb{N}$. Define

$$
\begin{gathered}
a_{k}:=\mu\left(\frac{2 \pi k}{|C|}+s_{0}\right)-\mu\left(s_{0}\right) \\
=\sum_{d=1}^{k}\left[\mu\left(\frac{2 \pi d}{|C|}+s_{0}\right)-\mu\left(\frac{2 \pi(d-1)}{|C|}+s_{0}\right)\right]
\end{gathered}
$$

according to corollary 3.3

$$
=k n \operatorname{sign}(C) \text {. }
$$

Thus

$$
\begin{gathered}
i^{*} m([f])=\lim _{t \rightarrow \infty} \frac{\mu(t)}{t}= \\
\lim _{k \rightarrow \infty} \frac{a_{k}}{\frac{2 \pi k}{|C|}}=\frac{n \operatorname{sign} C|C|}{2 \pi}=\frac{n C}{2 \pi} \\
=\frac{-n \widetilde{\operatorname{Cal}}([f])}{2 \pi v \operatorname{vol}(M)}
\end{gathered}
$$

as claimed.

Finally, the case $C=0$ follows from the considerations of step 2. Since according to what we have seen, we can take a homotopy of the path $\widetilde{\gamma}_{s_{0}+t}$ such that no rotation in the fibers occurs (see equation (17)), so clearly the asymptotic nonlinear Maslov index is zero.

\section{References}

[1] Banyaga, A.,Sur la structure du groupe des difféomorphismes qui préservent une forme symplectique, comm. Math. Helv. 53:2 (1978), 174-227.

[2] Bavard, C. Longueur stable des commutateurs, L'Enseign. Math. 37:1-2 (1991), 109-150.

[3] Calabi, E., On the group of automorphisms of a symplectic manifold, in problems in analysis, 1-26. Princeton Univ. Press, 1970. 
[4] Entov,M.;Polterovich,L. Calabi quasimorphism and quantum homology. Int.math.Res.Not.2003 no.30,1635-1676.

[5] Givental A.B. Nonlinear generalization of the Maslov index. Theory of singularities and its applications, Adv. Soviet Math., vol,1,American Mathematical society, Rhode Island,1990,p.71-103.

[6] Givental A.B. The nonlinear Maslov index. Geometry of low dimentional manifolds,2(Durham.1989), 35-43. London Math.Soc.Lecture Note Ser., 151,Camebridge university press 1990.

[7] McDuff,D., Salamon, D., Introduction to symplectic topology, second edition, Oxford University Press, Oxford, 1998.

[8] Polterovich L. The geometry of the group of symplectic diffeomorphisms. Lectures in Mathematics ETH Zürich Birkhäuser Verlag, Basel, 2001.

[9] Thèret, David. Rotation numbers of Hamiltonian isotopies in complex projective spaces. Duke Math. J.94(1998), no. 1, 13-27.

[10] Weinstein,A. Cohomology of Symplectomorphism Groups and Critical Values of Hamiltonians. Math.Zeit., 201, 75-82, 1989. 